\title{
FACTORS ASSOCIATED WITH DAMAGE IN A COHORT OF SYSTEMIC LUPUS ERYTHEMATOSUS PATIENTS
}

Anna Beatriz Gomes Souza Duarte ${ }^{1, *}$, Elisa Guimarães Motta, Gabriellen Vitiello1 ${ }^{1}$, osé Pinto de Souza Neto ${ }^{1}$, Marilena Mesquita Leal Fernandes ${ }^{1}$, Mauro Goldfarb ${ }^{1}$, Adriana Danowski ${ }^{1}$

1. Hospital Federal dos Servidores do Estado, Rio de Janeiro (RJ), Brazil.

*Corresponding author: annabeatrizgsduarte@gmail.com

\section{BACKGROUND}

Accrual of irreversible organ damage is a major risk factor for death in systemic lupus erythematosus (SLE). The aim of the study wase to identify the factors associated with damage in a cohort of SLE patients.

\section{MATERIALS AND METHODS}

We performed a cross-sectional study in patients longitudinally followed in a SLE cohort, accordingly to a standard protocol and collected by a computerized database. The Systemic Lupus International Collaborating Clinics / American College of Rheumatology Damage Index (SLICC / ACR SDI) was used to assess damage annually. Clinical features, demographics, serology, medications and complications were assessed. Univariate analyses were carried out on each potential predictive factor with Pearson's correlation or the Fisher's test when appropriate. A regression model was constructed to examine the significant variables.

\section{RESULTS}

Five hundred and seventy-six patients were included, $94.1 \%$ were female, mean age $50 \pm 8$ years, $40.8 \%$ Caucasian. The mean SLICC at the time of cohort enter was 0.69 and evolved to 1.25 at the time of this analysis. Damage accrual was associated with age $(p=0.02)$, mycophenolate $(p=0.01)$, azathioprine $(p=0.02)$, warfarin $(p<0.01)$, hydroxychloroquine $(p=0.00)$, prednisone $(p=0.00)$, photosensitivity $(p=0.03)$, discoid rash $(p=0.03)$ and smoking $(p<0.01)$. After multivariate analysis, previous use of azathioprine $(p<0.01 ; \mathrm{OR}=2.31,95 \% \mathrm{Cl}=1.47-3.64)$, prednisone $(\mathrm{p}=0.01 ; \mathrm{OR}=1.73,95 \% \mathrm{Cl}=1.10-2.71)$, photosensitivity $(p=0.03 ; O R=1.59,95 \% \mathrm{Cl}=1.03-2.44)$, age $(p=0.01 ; O R=2.33,95 \% \mathrm{Cl}=1.19-4.55)$, and smoking $(p=0.01 ; O R=1.74$, $95 \% \mathrm{Cl}=1.11-2.74)$ were associated with a higher risk of damage accrual. Mycophenolate $(p=0.01 ; \mathrm{OR}=0.50,95 \% \mathrm{Cl}=0.29-0.87)$ and hydroxychloroquine $(p=0.01 ; \mathrm{OR}=0.4895 \% \mathrm{Cl}=0.27-0.84)$ were protective.

\section{CONCLUSION}

As already identified in several multicenter studies, our work corroborates with the benefits of using hydroxychloroquine and mycophenolate mofetil in the treatment of patients with SLE: among several benefits they prevent damage. On the other hand, in our population, azathioprine is not effective in preventing future damage and we should carefully rethink its management. A great effort has to be done to use fewer steroids and stop smoking. These are habits that we can still modify and can be extremely important in preventing damage in SLE. 